\title{
Toward an Understanding of the Neural Circuitry of Major Depressive Disorder Through the Clinical Response to Deep Brain Stimulation of Different Anatomical Targets
}

\author{
Andrea L. Crowell • Patricio Riva-Posse • \\ Steven J. Garlow • Helen S. Mayberg
}

Published online: 18 March 2014

(C) Springer International Publishing AG 2014

\begin{abstract}
Deep brain stimulation has been proposed as a treatment for treatment-resistant depression. To date, multiple brain targets have been tested in single case studies and case series. The target regions with the most evidence to support their use are the subcallosal cingulate, ventral capsule/ventral striatum, nucleus accumbens, and medial forebrain bundle. Treatment effects of stimulation at each target share some commonalities, including similar response and remission rates, a relatively slow and progressive time course of treatment response, with a comparatively fast depressive relapse after discontinuation of stimulation. Response is maintained over time, and in some cases improves over years. Similarities may be at least partially attributable to overlap in white matter pathways between targets. Careful attention to mood and somatic effects seen with acute stimulation may differentiate primary vs. secondary treatment effects and mechanisms between the target regions. Understanding which symptoms are primarily affected, and the time course of response and relapse, will shed light on the biological mechanisms underlying DBS effects and depression pathology.
\end{abstract}

Keywords Deep brain stimulation · Functional neurosurgery · Depression · Subcallosal cingulate · Ventral capsule/ventral striatum - Nucleus accumbens · Medial forebrain bundle . Lateral habenula $\cdot$ Neurocircuitry

\section{Introduction}

Major Depressive Disorder (MDD) is increasingly recognized as a multidimensional illness with a complex underlying

A. L. Crowell $(\bowtie) \cdot$ P. Riva-Posse $\cdot$ S. J. Garlow $\cdot$ H. S. Mayberg

Department of Psychiatry and Behavioral Sciences, Emory

University School of Medicine, Atlanta, Georgia

e-mail: andrea.crowell@emory.edu circuitry responsible for the breadth of depressive symptoms. Neuromodulation techniques target circuit function to alleviate symptoms. Deep brain stimulation (DBS) has been shown to be an effective treatment in certain neurological disorders with known circuit dysfunction, notably movement disorders. Given this success, DBS has been put forward as a possible treatment for the estimated $10 \%$ of patients who suffer from treatment-resistant depression (TRD) [1]. An obvious first requirement is the selection of potentially relevant targets for stimulation. Complementary lines of reasoning have led to several candidate targets for testing of DBS for TRD. These strategies are outlined below.

\section{Role of Neuroimaging}

Several lines of evidence suggest involvement of corticostriatothalamocortical (CSTC) circuits in the pathophysiology of depression. Structural and functional abnormalities in several regions of this circuit have been observed in MDD patients. Volumetric abnormalities have been observed in the subgenual cingulate (SCC), dorsolateral prefrontal cortex (DLPFC), and hippocampus [2-4]. Tract tracing studies performed in non-human primates demonstrate connections from medial prefrontal regions to visceromotor output regions such as the hypothalamus and periaqueductal gray, to limbic areas including the amygdala and nucleus accumbens, and to midline thalamic structures [5-8].

Functional neuroimaging studies have demonstrated abnormal activity in several regions in this circuit in MDD patients, including over-activity in the subcallosal cingulate (BA 25) and amygdala, while under-activity is observed in the DLPFC, nucleus accumbens (NAC), and ventral striatum [9-12]. These abnormalities often correct with antidepressant treatment $[11,13,14]$. Abnormal NAC function is associated with abnormal reward processing, which may underlie 
anhedonia [15-17]. Dysregulation of the lateral habenula (LHb) may contribute to depressive symptoms through its role in processing negative feedback and error detection [18].

Target selection [Fig. 1] has been guided by these anatomical and functional abnormalities associated with MDD, as well as by observations of decreased depression symptoms following DBS treatment for OCD [19, 20], and past experience with ablation of specific white matter bundles [21, 22]. (For review, see Cosgrove, et al 2003 [23].) The three target sites with which there is the most DBS experience, while anatomically distinct, have shown promising results in several case series. Here we summarize findings from DBS for TRD trials and case series, in an effort toward understanding the circuit pathology of MDD.

\section{Specific DBS Targets under Investigation}

Subcallosal Cingulate (SCC)

\section{Rationale}

The SCC was initially chosen as a target for DBS for depression because of its increased activity during sadness, overactivity in MDD, and normalization of this over-activity with antidepressant treatment $[11,24]$. Inhibiting pathological over-activity at this target is hypothesized to allow release from a chronic pathological negative mood state. Modulation of the SCC is also capable of influencing other symptoms of depression, given how highly interconnected it is to regions involved in the expression of non-mood symptoms of depression. Connections with hypothalamus, brainstem, and insula make it possible for a dysfunctional SCC to modulate neurovegetative symptoms. Reciprocal connections with medial prefrontal, orbitofrontal, anterior and posterior cingulate, and subcortical structures such as the nucleus accumbens mediate SCC influence over cognitive, hedonic, and motivational symptoms of depression $[3,6,8]$.

\section{Results to date}

Six subjects were treated with DBS of the white matter adjacent to Brodmann Area 25 in 2005 [26]. Since then, results from a total of 77 patients (including seven with bipolar disorder type II) from eight clinical sites have been published [27, 28, 29•, 30-34]. Most studies involved open label stimulation; one included a single blind placebo lead-in month, as well as a limited blinded discontinuation phase that was halted due to clinical concerns regarding slow recovery of previously achieved clinical response with reactivated stimulation after several weeks [30].

Across studies, clinical response was defined as $50 \%$ reduction in the 17-item Hamilton Depression Rating Scale (HDRS-17) and remission as HDRS-17<8. Combined results averaged across studies show response rates of: $53 \%$ at six months, $47 \%$ at one year, $69 \%$ at two years, and $60 \%$ at three years; with remission rates of $27 \%$ at six months, $30 \%$ at one year, $39 \%$ at two years, and $40 \%$ at three years. [Table 1] Most of these study participants were assessed for up to one year following implantation. Holtzheimer et al reported on outcomes at two years for 12 subjects [30]. Kennedy and colleagues described 14 patients with between three to six years of follow-up [29•]. Response and remission rates are not only maintained, they continue to improve, underscoring long term efficacy of this treatment.

While a large scale, multi-center randomized sham controlled clinical trial has yet to be completed, the above studies
Fig. 1 Approximate targets for DBS for Major Depressive Disorder

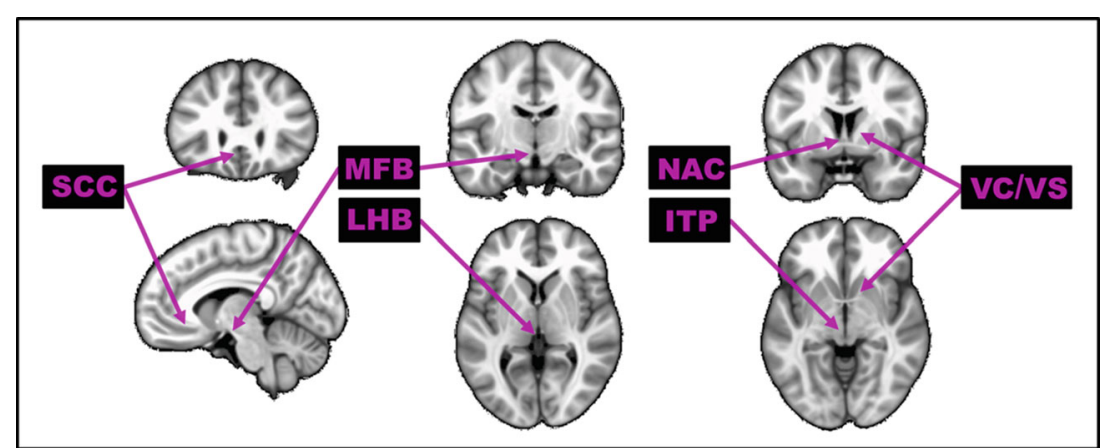

Putative depression targets for DBS. Figure adapted and updated from Holtzheimer and Mayberg, 2011 [25]. SCC = subcallosal cingulate, $\mathrm{VC} / \mathrm{VS}=$ ventral capsule /ventral striatum, NAC = nucleus accumbens, $\mathrm{MFB}=$ medial forebrain bundle, $\mathrm{LHb}=$ lateral habenula, $\mathrm{ITP}=$ inferior thalamic peduncle. 
Table 1 Summary of Deep Brain Stimulation for Depression Case Studies and Series

\begin{tabular}{|c|c|c|c|c|}
\hline Authors, Year & Target & Number & Follow up & Response/Remission \\
\hline Mayberg et al, 2005 [26] & SCC & 6 & 6 months & 6 months: $60 \% / 35 \%$ \\
\hline Lozano et al, 2008 [27] & SCC & 20 (incl. above 6) & 12 months & 12 months: $55 \% / 15 \%$ \\
\hline Kennedy et al, 2011 [29•] & $\mathrm{SCC}$ & 20 (same as above) & $>3$ years & $\begin{array}{l}2 \text { years: } 45 \% / 20 \% \\
3 \text { years: } 60 \% / 40 \%\end{array}$ \\
\hline Guinjoan et al, 2010 [28] & $\mathrm{SCC}$ & 1 & $>12$ months & Remission with unilateral stimulation \\
\hline Holtzheimer et al, 2012 [30] & $\mathrm{SCC}$ & 17 (7 bipolar) & 2 years & $\begin{array}{l}6 \text { months: } 41 \% / 18 \% \\
12 \text { months: } 36 \% / 36 \% \\
2 \text { years: } 92 \% / 58 \%\end{array}$ \\
\hline Puigdemont et al, 2011 [33] & SCC & 8 & 12 months & $\begin{array}{l}6 \text { months: } 88 \% / 38 \% \\
12 \text { months: } 63 \% / 50 \%\end{array}$ \\
\hline Lozano et al, 2012 [31] & SCC & 21 (multisite) & 12 months & $\begin{array}{l}6 \text { months: } 48 \% / \text { not reported } \\
12 \text { months: } 29 \% / \text { not reported }\end{array}$ \\
\hline Ramasubbu et al, 2013 [34] & SCC & 4 & 6 months & $50 \% / 0$ \\
\hline Merkl et al, 2013 [32] & $\mathrm{SCC}$ & 6 & 6 months & $33 \% / 33 \%$ \\
\hline Malone et al, 2009 [40] & $\mathrm{VC} / \mathrm{VS}$ & 15 (multisite, 1 bipolar) & 6-51 months & $\begin{array}{l}6 \text { months: } 47 \% / 20 \% \\
\text { LFU: } 53 \% / 40 \%\end{array}$ \\
\hline Schlaepfer et al, 2008 [45] & NAC & 3 & 6-22 weeks & Response at one week \\
\hline Bewernick et al, 2010 [47] & NAC & 10 (Incl. above 3) & 12 months & $50 \% / 30 \%$ \\
\hline Bewernick et al, 2012 [46] & NAC & 11 (Incl. above 10) & 2 years & $45.5 \% / 45.5 \%$ \\
\hline Schlaepfer et al, 2013 [50] & MFB & 7 (1 bipolar) & $12-33$ weeks & 12 weeks: $86 \%$ / $57 \%$ \\
\hline Sartorius et al, 2010 [58] & $\mathrm{LHb}$ & 1 & $>50$ weeks & Remission \\
\hline Jimenez et al, 2005 [52] & ITP & 1 & 24 months & Remission \\
\hline
\end{tabular}

$\mathrm{SCC}=$ subcallosal cingulate, $\mathrm{VC} / \mathrm{VS}=$ ventral capsule $/$ ventral striatum, $\mathrm{NAC}=$ nucleus accumbens, $\mathrm{MFB}=$ medial forebrain bundle, $\mathrm{LHb}=$ lateral habenula, ITP = inferior thalamic peduncle, $\mathrm{LFU}=$ last follow up

demonstrate a consistent pattern and timeline of sustained behavioral effects in multiple independent patient samples. Interestingly, these similar results are seen despite clear differences in inclusion/exclusion criteria, methods for surgical targeting, and algorithms for contact selection and parameter adjustments - all potentially critical variables in optimizing the procedure. To date, significant effort has gone into defining the ideal SCC white matter target and refining the surgical procedure. Traditional anatomical targeting has not clearly identified responder vs. non-responder target regions $[32,35]$. New evidence demonstrates that the optimal target includes the intersection and stimulation of three independent tracts that meet at the mid-subcallosal cingulate - the uncinate fasciculus, cingulum bundle, and forceps minor $\left[36^{\bullet}\right]$.

\section{Adverse Events}

Surgical and Device-Related Like all neurosurgical interventions, complications of DBS can include stroke, seizure, hemorrhage, and adverse effects of anesthesia. Postoperatively, pain is common and infections are possible. Device-related complications such as broken leads or extension cables, battery failure, and scar tissue formation may occur. These potential adverse outcomes could be expected with any surgical implantation of a foreign device, and given the relatively small numbers of studies to date, there is insufficient data to detect any differences that might exist between devices or surgical targets. Outside of surgery, adverse effects of DBS treatment may reflect device failure, stimulation-induced symptoms, therapeutic non-response or loss of therapeutic effect.

At the SCC target, device explantation due to infection with subsequent reimplantation has been described in two subjects $[29 \bullet, 30]$. Three patients were explanted due to lack of benefit within two years of implantation [29•].

Intraoperative and Acute Stimulation Positive effects of DBS stimulation during the intraoperative procedure have been documented, and include subject reports of increased calmness and interest [26, 27], sudden lightness, awareness, connectedness, and "disappearance of the void"[26]. Intraoperative response has been used to guide initial contact selection, but has not been reported to be predictive of long- 
term antidepressant effect. In contrast to other stimulation targets, stimulation parameter adjustments do not typically cause acute changes in emotional or physical symptoms. There were no documented cases of mania or hypomania related to stimulation changes.

Discontinuation Worsening of depression symptoms consistently occurs with intended or unintended discontinuation. Unintended discontinuations due to battery depletion, accidental turn off, or other device failure are described in several studies and are often associated with loss of antidepressant effect, even after years of chronic stimulation. The return of depression symptoms is usually subacute, occurring over one to six weeks $[26,27,29 \bullet, 30]$. Notably, there are no rapid changes in negative mood with acute discontinuation, allowing medical tests such as an EKG to be performed without risk.

\section{Ventral Capsule/Ventral Striatum (VC/VS)}

\section{Rationale}

Selection of this target for DBS for depression grew largely out of historical precedent. Early surgical interventions for treatment-resistant depression and obsessive-compulsive disorder (OCD) included anterior cingulotomy, anterior capsulotomy, subcaudate tractotomy, and limbic leucotomy. The precedent of high frequency stimulation as a reversible lesion for patients with movement disorders provided a plausible DBS strategy for patients with intractable psychiatric disorders.

The earliest DBS studies for psychiatric conditions targeted the anterior limb of the internal capsule for treatment of OCD [37]. These studies found that DBS to this region improved symptoms of OCD as well as comorbid depression symptoms $[19,20,38]$. It was suggested that axons passing through this region of the anterior internal capsule to the OFC were responsible for the beneficial effects of DBS on OCD [19]. This region of the anterior internal capsule also contains fiber tracts connecting the subgenual cingulate to the thalamus [39], while the adjacent ventral striatum includes regions linked to motivation and reward. The antidepressant effects seen in the OCD experience, and anatomical evidence linking VC/VS with structures relevant to depression symptoms, support the use of the $\mathrm{VC} / \mathrm{VS}$ target for MDD.

Results to date

Malone and colleagues report on the results of fifteen patients (14=MDD, $1=$ bipolar type I) who were implanted in the ventral capsule / ventral striatum (VC/VS) at three clinical sites [40]. The primary outcome measure was the 24-item
Hamilton Depression Rating Scale, with a $50 \%$ reduction in score considered response and remission defined as a score $\leq$ 10. Response rates were $50 \%$ at three months and $47 \%$ at six months. Remission rates were $20 \%$ at three months and six months. The mean follow up period was $23.5( \pm 14.9)$ months and response and remission rates at the time of last follow up were $53 \%$ and $33 \%$, respectively [Table 1]. Five of the 15 patients had at least three years of follow-up (range 3651 months). Of these: $60 \%$ were responders and $40 \%$ were in remission at the time of last follow up. A larger scale multicenter clinical trial was conducted but definitive results have not been published.

\section{Adverse Events}

Surgical and Device-Related Two subjects required surgical revision due to a lead break and a problem with the extension cable.

Intraoperative and Acute Stimulation Response to intraoperative stimulation occurred in some patients, who described improved mood, decreased anxiety, and increased awareness. Acute adverse effects included increased anxiety, sweating, speech perseveration, and facial motor effects. These effects helped to guide contact selection for chronic stimulation, but do not appear to predict treatment response. There were two episodes of hypomania in the bipolar patient, which resolved with modification of medication and stimulation parameters. One episode of hypomania occurred in a patient with MDD, which resolved with stimulation parameter changes.

Discontinuation Three incidents of increased depression were attributed to battery depletion or accidental turn off of the device. Depression symptoms after discontinuation of stimulation "returned quickly in some patients," although no specific time course is reported.

\section{Nucleus Accumbens (NAC)}

\section{Rationale}

A key line of reasoning for DBS to the NAC for depression is the conceptualization of anhedonia as a defining symptom of depression and the characterization of anhedonia as the lack of reward-motivated behavior. Multiple lines of evidence support a central role for the nucleus accumbens in mediating drive and motivation, as well as normal reward processing and reward-seeking behaviors. There is evidence of abnormal reward processing in patients with depression, and this dysfunctional processing is associated with abnormal activity in the ventral striatum, as reviewed by Eshel and Roiser [41]. The nucleus accumbens sends and receives projections from 
brain regions that are involved in emotional processing, the motor system, memory, and dopamine production. Thus, beyond hypothesized direct effects on reward processing and motivation, the NAC is positioned to affect brain regions involved in other symptoms of the depressive syndrome. Interestingly, the SCC target projects directly to the shell of the accumbens [42], providing a potential point of convergence across the two targets. However, direct stimulation within a nucleus is likely producing different effects than stimulation of efferent projections from the cingulate and medial frontal cortex targeting by the subcallosal cingulate target. That the impact of the two approaches might be different is suggested from recent animal studies examining optogenetic stimulation of the infralimbic cortex versus the nucleus accumbens and associated fiber bundles $[43 \bullet, 44]$.

\section{Results to date}

Eleven cases of DBS to the NAC have been reported [45-47]. The primary outcome measure was the 28 -item Hamilton Depression Rating Scale (HDRS-28), with response considered a $50 \%$ score reduction and remission a score of $<10$. Five of the 11 patients $(45.5 \%$ ) were responders and $30 \%$ of subjects met remission criteria at one year [Table 1]. Remission was maintained at two years in only one subject, while in those who responded at 12 months, response was maintained for up to four years. Responders and nonresponders descriptively differed in depression ratings from one month of stimulation onward. There were no cases in which a treatment non-responder at 12 months became a responder later.

\section{Adverse Events}

Surgical and Device-Related There was one case of device explantation after two years due to an infection acquired during chemotherapy treatment for cancer.

Intraoperative and Acute Stimulation Subjects reported being unable to detect whether stimulation was on or off. However, near immediate changes in behavior that were suggestive of increased pleasure-seeking were reported with acute stimulation, along with a significant drop in depression scores. Erythema, anxiety, sweating, paresthesias, and hypomania were reported as side effects of acute stimulation; psychotic symptoms were reported in a single case. All resolved with a change in stimulation parameters. Stimulation setting changes were sometimes associated with acute mood improvement lasting up to two weeks, but these episodes were not predictive of long term outcome.

Discontinuation Blinded discontinuation after one week of stimulation was halted due to acute and significant worsening of symptoms. The effects of stimulation on depression scores were described as "immediate and bidirectional," with improvement and worsening of depression scores as soon as stimulation was turned on or off [45]. Similarly, accidental discontinuation, such as with battery depletion, also resulted in "rapid" return of depression symptoms [47].

\section{Medial Forebrain Bundle (MFB)}

\section{Rationale}

In line with the hypothesis that modulation of reward system circuitry would effectively treat anhedonia and depression, evidence from animal and imaging studies suggested the superolateral branch of the medial forebrain bundle (slMFB) as a potential target. The medial forebrain bundle connects the ventral tegmental area (VTA) and NAC, crosses under the thalamus and rises to the anterior limb of the internal capsule, running parallel to the anterior thalamic radiation and projecting to the orbitofrontal cortex and dorsolateral prefrontal cortex. It can inconsistently be seen connecting to white matter fibers surrounding the SCC [48] and likely depends on the current used as to whether it reaches or not. Further, mood effects with inadvertent stimulation of MFB may be seen, as hypomania with subthalamic nucleus stimulation in Parkinson's disease has been causally linked to current spread to MFB [49]. Electric field modeling performed by the same group suggests that current spread from stimulation of any of the above targets (SCC; VC/VS; NAC) may reach the slMFB. It is important to note that, while these regions are indeed connected, information flow is not uniform, and systematic studies of differences between afferent and efferent fibers would greatly inform understanding of this issue.

\section{Results to date}

Results of MFB DBS have been described in seven subjects ( $6=$ MDD, 1 = bipolar type I) observed for up to 33 weeks [50]. Response was defined as $50 \%$ reduction in the Montgomery- Åsberg Depression Rating Scale (MADRS). Rapid reduction in depression symptoms was reported in six of seven subjects within two days of initiation of chronic stimulation. Study response criteria were met in four of seven subjects at week one, and five of seven subjects after six weeks. In most subjects, once response was achieved, it was maintained. At 12 weeks, six of seven subjects were classified as responders, and four of those were classified as remitters, demonstrating a MADRS score of $<10$ [Table 1]. These numbers remained the same at the time of last follow up (12-33 weeks). Of note, the HDRS-28 was used as a secondary outcome measure, and at 12 weeks only two of seven subjects experienced $\geq 50 \%$ score reduction on this scale. 


\section{Adverse Events}

Surgical and Device-Related One patient experienced a small intracranial hemorrhage at the intended target site, with transient hemiparesis and dysarthria. Interestingly, the authors hypothesize that the resulting damage prevented DBS response in this non-responder subject [51].

Intraoperative and Acute Stimulation All seven subjects demonstrated acute intraoperative effects, including initiating eye contact, engaging in conversation, and reporting improved mood. Stimulation parameters were limited by blurred vision and strabismus at higher stimulation amplitudes on specific electrode contacts, which were experienced by all subjects. Stimulation-related dizziness and sweating were also reported. No hypomania was reported.

Discontinuation Discontinuation of stimulation was not described in this short duration study.

\section{Single Case Reports}

\section{Inferior Thalamic Peduncle}

Jimenez and colleagues [52] suggested the use of the inferior thalamic peduncle (ITP) as a target for the surgical treatment of depression. They describe the role of ITP fibers in connecting midline and intralaminar thalamic nuclei of the non-specific thalamic system with the orbital frontal cortex. They proposed that the ITP is part of an inhibitory system connecting the OFC to subcortical structures, in particular to the thalamus.

A patient with chronic MDD and comorbid borderline personality disorder and bulimia underwent bilateral ITP implantation. This produced remission of her depressive symptoms. Discontinuation of stimulation resulted in a return of some mild depression symptoms, which remitted with resumption of stimulation.

\section{Lateral Habenula}

Sartorius and Henn [53] made a cohesive argument for the use of the lateral habenula $(\mathrm{LHb})$ as an antidepressant DBS target. The lateral habenula receives limbic and cortical afferents, including from the inferior thalamic peduncle targeted by Jimenez et al, and has serotonergic, noradrenergic, and dopaminergic projections to the dorsal raphe, locus coeruleus, and VTA, putting it in a position to influence neurotransmitter systems modulated by current pharmacotherapies and other brain regions implicated in depression symptoms. Convergent lines of evidence support a link between LHb hyperactivity and depressed mood states. In multiple animal models, elevated activity in the $\mathrm{LHb}$ was associated with depressionlike behaviors, which was blocked by monoamine oxidase inhibitor treatment [54-56]. The LHb sends efferents to the VTA and stimulation of the habenula inhibits dopamine neurons there [55]. In healthy subjects, habenula activation occurs when an error in reward prediction is made, particularly when negative feedback about the error is received [18]. Overactivity thus may indicate heighted sensitivity to negative reward feedback in the environment, or a false positive error detection signal, and contributes to decreased reward signaling via its inhibition of VTA dopamine neurons. LHb activity has also been shown to be inversely related to the incentive salience of a conditioned reward cue [57]. Dulled incentive salience may contribute to the amotivation and abnormal reward processing seen in depression.

Bilateral DBS to the LHb was performed in one patient with MDD [58]. Her depressive episode remitted with stimulation, and she remained in remission until her device was accidentally (and unknown to the patient) turned off, which resulted in prompt return of depressive symptoms. Three months after stimulation was reinstated at the previously effective settings her depression again remitted. A clinical trial is open for recruitment (clinicaltrials.gov NCT01798407).

\section{Conclusion}

Deep brain stimulation for depression shows promising results in a population of highly treatment-resistant patients. Encouraging outcomes have been reported for the SCC, VC/ VS, and NAC/MFB brain targets, which share broadly similar clinical effects. Acute behavioral changes or improved mood occur in some patients when stimulation is turned on in the operating room, although these acute stimulation effects do not necessarily predict chronic response to stimulation. Stimulation-related adverse effects, such as hypomania and sweating were more common in the NAC and MFB and were not seen in the SCC. Improvement in depressive symptoms typically occurs over months, although MFB stimulation appears to work more rapidly. Response to stimulation is sustained, and in some cases improves, over years. Across all targets there is significant worsening of symptoms when stimulation is discontinued, albeit with distinctly different timelines.

The similarities in response to chronic stimulation across these distinctly different anatomical locations invites the hypothesis that all targets are nodes within a larger distributed neural network, where stimulation at any individual node results in similar net behavioral effects. All of the brain regions currently targeted in depression are connected to multiple other areas implicated in depression pathology, and there is significant overlap in connectivity patterns across targets [59]. Further, there is also growing evidence that targeting specific 
combinations of white matter tracts, rather than specific grey matter nuclei, is necessary for antidepressant response to DBS $\left[36 \bullet, 60^{\bullet}\right]$. Regardless of target, an understanding of the white matter tracts that are necessary and sufficient for long-term antidepressant response is crucial. Even DBS to the NAC undoubtedly includes within its stimulation field fibers of passage that may be relevant to its therapeutic effect.

Differences between targets provide an opportunity to better understand DBS mechanisms and primary versus secondary treatment effects. While intraoperative responses have been documented across targets, there are notable differences in the nature and degree of the response to acute stimulation, both in the operating room and in the clinic. These acute effects may reflect primary mechanisms by which DBS is acting at a given target. With SCC DBS, subjects have reported increased vividness of the environment and increased sense of calm, but these effects are not reliably replicated with repeated testing in the clinic and do not predict long-term antidepressant response. In contrast, improvement in depression ratings can be seen acutely in the NAC/MFB and may last up to two weeks following parameter changes. However, this improvement may subside and is not predictive of long-term chronic response [47]. It is unclear whether the same pathways mediate both the acute behavioral changes and those that occur more gradually in patients who become treatment responders.

Careful characterization of the nature and time course of mood and behavior changes may reveal differences in primary versus secondary therapeutic effects of stimulation at different targets. SCC DBS appears to primarily target negative mood. Lifting negative mood in turn appears to enable the capacity for re-establishing new skills and patterns of behavior, with secondary effects on motivation and reinforcement. DBS to the VC/VS, NAC, and MFB, on the other hand, may target anhedonia by acting more directly on primary drive systems. Increased motivation and enjoyment then allows for the ability to engage in activities that promote euthymia.

Long-term results of SCC DBS show improvement that is relatively slow but progressive. Treatment response typically develops over months, but continues to accrue over years. In contrast, the antidepressant response to MFB DBS occurs more quickly. This response is maintained over time, but has not yet been shown to improve over time, as long-term results are not yet reported. Notably, an acute to subacute return of depressive symptoms follows discontinuation of stimulation across all targets, but at distinctly different rates. The predictability of this symptomatic relapse raises questions about the fundamental biological effects of DBS. The reliable return of depression symptoms, even after years of chronic stimulation, suggests that DBS may serve a "holding" function in the network that prevents reversion to the prior pathological state. A more gradual return of symptoms, as is seen at the SCC target might suggest that the network has developed a degree of resilience to inherent pathological tendencies, possibly owing to plasticity. Anecdotally, despite the return of depressive symptoms, the net change still remains a net improvement over pre-surgical symptom severity, suggesting some form of neuroplasticity as an important mediator of the longterm benefit of DBS.

Next steps will require systematic studies in larger cohorts of patients to better characterize the differential time course of response, sustainability of effects, relapse potential with discontinuation; and importantly, subtle differences in maximally improved symptoms across the different DBS targets. Such comparisons may eventually prove useful in choosing among the available options for a given patient. Future studies will also continue to address refinement of surgical targeting strategies and mechanisms of symptom improvement, to best optimize the safety and effectiveness of DBS for depression, regardless of the approach.

\section{Compliance with Ethics Guidelines}

Conflict of Interest Andrea Crowell has a pending fellowship award from APA/Lilly Psychiatric Fellowship. Helen S. Mayberg has a consulting agreement with St Jude Medical Inc., which has licensed her intellectual property to develop SCC DBS for the treatment of severe depression. Patricio Riva-Posse has no outside interests or conflicts to report. Steven J. Garlow has no outside interests or conflicts to report. DBS research supported by grants from NARSAD, Dana Foundation, Stanley Medical Research Institute, Woodruff Foundation, Hope for Depression Research Foundation.

Human and Animal Rights and Informed Consent This article does not contain any studies with human or animal subjects performed by the author.

\section{References}

Papers of particular interest, published recently, have been highlighted as:

- Of importance

1. Holtzheimer PE, Mayberg HS. Stuck in a rut: rethinking depression and its treatment. Trends Neurosci. 2011;34(1):1-9.

2. Rajkowska $\mathrm{G}$ et al. Morphometric evidence for neuronal and glial prefrontal cell pathology in major depression. Biol Psychiatry. 1999;45(9):1085-98.

3. Ongur D, Drevets WC, Price JL. Glial reduction in the subgenual prefrontal cortex in mood disorders. Proc Natl Acad Sci U S A. 1998;95(22):13290-5.

4. Gittins RA, Harrison PJ. A morphometric study of glia and neurons in the anterior cingulate cortex in mood disorder. J Affect Disord. 2011;133(1-2):328-32.

5. Ongur D, Price JL. The organization of networks within the orbital and medial prefrontal cortex of rats, monkeys and humans. Cereb Cortex. 2000;10(3):206-19. 
6. Barbas $\mathrm{H}$ et al. Serial pathways from primate prefrontal cortex to autonomic areas may influence emotional expression. BMC Neurosci. 2003;4:25.

7. Petrides M, Pandya DN. Dorsolateral prefrontal cortex: comparative cytoarchitectonic analysis in the human and the macaque brain and corticocortical connection patterns. Eur J Neurosci. 1999;11(3): 1011-36.

8. Haber SN. The primate basal ganglia: parallel and integrative networks. J Chem Neuroanat. 2003;26(4):317-30.

9. Baxter Jr LR et al. Reduction of prefrontal cortex glucose metabolism common to three types of depression. Arch Gen Psychiatry. 1989;46(3):243-50.

10. Siegle GJ et al. Increased amygdala and decreased dorsolateral prefrontal BOLD responses in unipolar depression: related and independent features. Biol Psychiatry. 2007;61(2):198-209.

11. Mayberg HS et al. Reciprocal limbic-cortical function and negative mood: Converging PET findings in depression and normal sadness. Am J Psychiatr. 1999;156(5):675-82.

12. Epstein $\mathrm{J}$ et al. Lack of ventral striatal response to positive stimuli in depressed versus normal subjects. Am J Psychiatry. 2006;163(10): 1784-90.

13. Fales CL et al. Antidepressant treatment normalizes hypoactivity in dorsolateral prefrontal cortex during emotional interference processing in major depression. J Affect Disord. 2009;112(1-3):20611 .

14. Mayberg HS. Targeted electrode-based modulation of neural circuits for depression. J Clin Invest. 2009;119(4):717-25.

15. Pizzagalli DA, Jahn AL, O'Shea JP. Toward an objective characterization of an anhedonic phenotype: a signal-detection approach. Biol Psychiatry. 2005;57(4):319-27.

16. Hall GB, Milne AM, Macqueen GM, An fMRI study of reward circuitry in patients with minimal or extensive history of major depression. Eur Arch Psychiatry Clin Neurosci, 2013.

17. Wacker J, Dillon DG, Pizzagalli DA. The role of the nucleus accumbens and rostral anterior cingulate cortex in anhedonia: integration of resting EEG, fMRI, and volumetric techniques. Neuroimage. 2009;46(1):327-37.

18. Ullsperger M, von Cramon DY. Error monitoring using external feedback: specific roles of the habenular complex, the reward system, and the cingulate motor area revealed by functional magnetic resonance imaging. J Neurosci. 2003;23(10):4308-14.

19. Abelson JL et al. Deep brain stimulation for refractory obsessivecompulsive disorder. Biol Psychiatry. 2005;57(5):510-6.

20. Nuttin BJ et al. Long-term Electrical Capsular Stimulation in Patients with Obsessive-Compulsive Disorder. Neurosurgery. 2003;52(6):1263-74.

21. Shields DC et al. Prospective assessment of stereotactic ablative surgery for intractable major depression. Biol Psychiatry. 2008;64(6):449-54.

22. Steele JD et al. Anterior cingulotomy for major depression: clinical outcome and relationship to lesion characteristics. Biol Psychiatry. 2008;63(7):670-7.

23. Cosgrove GR, Rauch SL. Stereotactic cingulotomy. Neurosurg Clin N Am. 2003;14(2):225-35.

24. Mayberg HS et al. Regional metabolic effects of fluoxetine in major depression: serial changes and relationship to clinical response. Biol Psychiatry. 2000;48(8):830-43.

25. Holtzheimer PE, Mayberg HS. Deep brain stimulation for psychiatric disorders. Annu Rev Neurosci. 2011;34:289-307.

26. Mayberg HS et al. Deep brain stimulation for treatment-resistant depression. Neuron. 2005;45(5):651-60.

27. Lozano AM et al. Subcallosal cingulate gyrus deep brain stimulation for treatment-resistant depression. Biol Psychiatry. 2008;64(6): 461-7.

28. Guinjoan SM et al. Asymmetrical contribution of brain structures to treatment-resistant depression as illustrated by effects of right subgenual cingulum stimulation. J Neuropsychiatry Clin Neurosci. 2010;22(3):265-77.

29. Kennedy SH et al. Deep brain stimulation for treatment-resistant depression: follow-up after 3 to 6 years. Am J Psychiatry. 2011;168(5):502-10. This study is notable as it reports the longest follow up to date of DBS for depression. Twenty patients were followed for three to six years. Intent to treat analysis shows $60 \%$ response rate and $40 \%$ remission rate with three years of chronic stimulation to the subcallosal cingulate.

30. Holtzheimer PE et al. Subcallosal cingulate deep brain stimulation for treatment-resistant unipolar and bipolar depression. Arch Gen Psychiatry. 2012;69(2):150-8.

31. Lozano AM et al. A multicenter pilot study of subcallosal cingulate area deep brain stimulation for treatment-resistant depression. $\mathrm{J}$ Neurosurg. 2012;116(2):315-22.

32. Merkl A et al. Antidepressant effects after short-term and chronic stimulation of the subgenual cingulate gyrus in treatment-resistant depression. Exp Neurol. 2013;249:160-8.

33. Puigdemont D, et al. Deep brain stimulation of the subcallosal cingulate gyrus: further evidence in treatment-resistant major depression. Int J Neuropsychopharmacol, 2011; 1-13.

34. Ramasubbu R et al. Double-blind optimization of subcallosal cingulate deep brain stimulation for treatment-resistant depression: a pilot study. J Psychiatry Neurosci. 2013;38(5):325-32.

35. Hamani $\mathrm{C}$ et al. Deep brain stimulation of the subcallosal cingulate gyrus for depression: anatomical location of active contacts in clinical responders and a suggested guideline for targeting. $\mathrm{J}$ Neurosurg. 2009;111(6):1209-15.

36. Riva-Posse $P$ et al. Optimization of subcallosal cingulate deep brain stimulation for treatment-resistant depression using structural connectivity. Biol Psychiatry. 2013;73(9, Supplement):A1-8. 1S344S.This study demonstrates there are three separate white matter tracts that are necessary for antidepressant response to subcallosal cingulate DBS.

37. Nuttin B et al. Electrical stimulation in anterior limbs of internal capsules in patients with obsessive-compulsive disorder. Lancet. 1999;354(9189): 1526.

38. Aouizerate B et al. Deep brain stimulation of the ventral caudate nucleus in the treatment of obsessive-compulsive disorder and major depression. J Neurosurg. 2004;101(4):682-6.

39. Hsu DT, Price JL. Midline and intralaminar thalamic connections with the orbital and medial prefrontal networks in macaque monkeys. J Comp Neurol. 2007;504(2):89-111.

40. Malone Jr DA et al. Deep brain stimulation of the ventral capsule/ ventral striatum for treatment-resistant depression. Biol Psychiatry. 2009;65(4):267-75.

41. Eshel N, Roiser JP. Reward and punishment processing in depression. Biol Psychiatry. 2010;68(2):118-24.

42. Ferry AT et al. Prefrontal cortical projections to the striatum in macaque monkeys: evidence for an organization related to prefrontal networks. J Comp Neurol. 2000;425(3):447-70.

43. Vassoler FM et al. Deep brain stimulation of the nucleus accumbens shell attenuates cocaine reinstatement through local and antidromic activation. J Neurosci. 2013;36:14446-54. Showed that DBS to the nucleus accumbens shell attenuated cocaine reinstatement, not by inactivating the target nucleus or fibers of passage, but through antidromic activation of inhibitory interneurons in the prefrontal cortex.

44. Warden MR et al. A prefrontal cortex-brainstem neuronal projection that controls response to behavioural challenge. Nature. 2012;492(7429):428-32.

45. Schlaepfer TE et al. Deep brain stimulation to reward circuitry alleviates anhedonia in refractory major depression. Neuropsychopharmacology. 2008;33(2):368-77.

46. Bewernick BH et al. Long-term effects of nucleus accumbens deep brain stimulation in treatment-resistant depression: evidence for 
sustained efficacy. Neuropsychopharmacology. 2012;37(9):197585 .

47. Bewernick BH et al. Nucleus accumbens deep brain stimulation decreases ratings of depression and anxiety in treatment-resistant depression. Biol Psychiatry. 2010;67(2):110-6.

48. Coenen VA et al. Cross-species affective functions of the medial forebrain bundle-implications for the treatment of affective pain and depression in humans. Neurosci Biobehav Rev. 2011;35(9):1971-81.

49. Coenen VA et al. Medial forebrain bundle stimulation as a pathophysiological mechanism for hypomania in subthalamic nucleus deep brain stimulation for Parkinson's disease. Neurosurgery. 2009;64(6):1106-14. discussion 1114-5.

50. Schlaepfer TE et al. Rapid effects of deep brain stimulation for treatment-resistant major depression. Biol Psychiatry. 2013;73(12): 1204-12.

51. Coenen VA, Madler B, Schlaepfer TE. Reply to: medial forebrain bundle stimulation-speed access to an old or entry into a new depression neurocircuit? Biol Psychiatry. 2013;74(12):e45-6.

52. Jimenez $\mathrm{F}$ et al. A patient with a resistant major depression disorder treated with deep brain stimulation in the inferior thalamic peduncle. Neurosurgery. 2005;57(3):585-93.

53. Sartorius A, Henn FA. Deep brain stimulation of the lateral habenula in treatment resistant major depression. Med Hypotheses. 2007;69(6):1305-8.
54. Shumake J, Edwards E, Gonzalez-Lima F. Opposite metabolic changes in the habenula and ventral tegmental area of a genetic model of helpless behavior. Brain Res. 2003;963(1-2):274-81.

55. Matsumoto M, Hikosaka O. Lateral habenula as a source of negative reward signals in dopamine neurons. Nature. 2007;447(7148): 1111-5.

56. Caldecott-Hazard S, Mazziotta J, Phelps M. Cerebral correlates of depressed behavior in rats, visualized using 14C-2-deoxyglucose autoradiography. J Neurosci. 1988;8(6):1951-61.

57. Danna CL, Shepard PD, Elmer GI. The habenula governs the attribution of incentive salience to reward predictive cues. Front Hum Neurosci. 2013;7:781.

58. Sartorius A et al. Remission of major depression under deep brain stimulation of the lateral habenula in a therapy-refractory patient. Biol Psychiatry. 2010;67(2):e9-e11.

59. Gutman DA et al. A tractography analysis of two deep brain stimulation white matter targets for depression. Biol Psychiatry. 2009;65(4):276-82.

60. Lujan JL et al. Axonal pathways linked to therapeutic and nontherapeutic outcomes during psychiatric deep brain stimulation. Hum Brain Mapp. 2012;33(4):958-68. Demonstrates five white matter tracts shared by most subjects who responded to DBS to the ventral capsule/ventral striatum. There was one white matter tract common to all tre + atment non-responders. 